\title{
Historia y futuro del mantenimiento y la vigilancia de los puentes ferroviarios
}

\section{Past and Future of Maintenance and Monitoring of Railway Bridges}

\author{
Luis Esteras Aldea y José Antonio Gómez Barquín
}

\author{
a Ingeniero de Caminos, Canales y Puertos. Gerente de Área de Infraestructura de la Dirección Técnica de ADIF \\ ${ }^{b}$ Ingeniero de Caminos, Canales y Puertos. Jefe de Puentes de la Dirección Técnica de ADIF
}

Recibido el 30 de noviembre de 2020; aceptado el 30 de marzo de 2021

\section{RESUMEN}

El mantenimiento de las infraestructuras ha venido ocupando frente a la construcción de obra nueva un segundo plano a lo largo de la historia de la ingeniería, siempre eclipsado por las facetas de diseño y construcción. No obstante, cada vez la conservación y el mantenimiento de las infraestructuras se va haciendo hueco en las escuelas técnicas, en la normativa y en las licitaciones públicas gracias al esfuerzo diario de los grandes profesionales que se dedican a ello. Se repasará cómo ha sido esta tendencia en el caso particular de los puentes ferroviarios.

PALABRAS ClAVE: Mantenimiento de infraestructuras, puente ferroviario, inspección principal, prueba de carga, normativa, monitorización de estructuras.

(C) 2021 Asociación Española de Ingeniería Estructural (ACHE). Publicado por Cinter Divulgación Técnica S.L. Todos los derechos reservados.

\section{ABSTRACT}

The maintenance of infrastructures has been in a secondary place throughout the history of engineering, always overshadowed by the design and construction of new bridges. However, conservation and maintenance of infrastructures is gaining positions in the technical schools, in regulations and in public tenders thanks to the daily effort of the great professionals who dedicate themselves to it. Let's evaluate the trend in the particular case of railway bridges.

KEYWORDS: Maintenance of infrastructures, railway bridge, main inspection, load test, regulations, structure monitoring.

(c) 2021 Asociación Española de Ingeniería Estructural (ACHE). Published by Cinter Divulgación Técnica S.L. All rights reserved.

1.

\section{INTRODUCCIÓN}

El desarrollo de la técnica de la ingeniería de caminos se ha orientado desde sus orígenes en la fase de construcción de obra nueva principalmente, y el mantenimiento de las infraestructuras construidas ha quedado relegado a un segundo plano, abandonado a la suerte de los técnicos que se han dedicado al mantenimiento. Lejos de desmerecer estas actividades de mantenimiento y reparación de estructuras es el momento de que se reconozca esta labor silenciosa que no corta cintas. Luis Ortega, al igual que otros muchos ingenieros dedicados a la

* Persona de contacto / Corresponding author:

Correo-e / email: lesteras@adif.es / joangomez@adif.es conservación y mantenimiento de las infraestructuras, ha sido un auténtico referente para aquellos que trabajamos en estas labores "olvidadas". Si echáramos cuentas de los importes dedicados a la construcción de obra nueva en comparación con lo dedicado al mantenimiento veríamos la diferencia que todavía hoy hay entre unos presupuestos y otros.

En lo que se refiere al desarrollo normativo la situación es muy similar, los esfuerzos se focalizan en determinar cómo deben construirse las nuevas estructuras habiendo muy poca normativa de referencia sobre cómo deben mantenerse esas mismas estructuras.

La infraestructura ferroviaria no es una excepción y presenta la misma situación que el resto de las obras civiles en este sentido. 
Afortunadamente en los últimos años se ha hecho un esfuerzo importante para desarrollar los diferentes documentos normativos que configuran la gestión de los puentes ferroviarios.

Con la entrada en vigor el 25 de junio de 2005 de la orden FOM/1951/2005 instrucción sobre las inspecciones técnicas en los puentes de ferrocarril (ITPF-05) [1] se sentaron las bases para la organización del mantenimiento de los puentes ferroviarios. Pero veamos cuál ha sido la evolución hasta ese momento.

\section{2.}

\section{REPASO HISTÓRICO}

Repasemos brevemente la historia sobre la normativa que regula la vigilancia de los puentes ferroviarios:

La primera norma que se publica relativa al diseño de puentes de ferrocarriles es la de 1902, instrucción para redactar proyectos de puentes metálicos [2], la cual ya nació desfasada en cuanto a las cargas de diseño, pero en cuanto a lo que en este artículo nos afecta, no se hace mención alguna a la vigilancia a realizar sobre los puentes, y debemos esperar hasta la norma de 1925, Instrucción para la redacción de proyectos de tramos metálicos [3], gracias fundamentalmente al magnífico trabajo desarrollado por Don Domingo Mendizábal, para tener una norma mucho más acorde a la realidad del momento y que como veremos, en cuanto a la vigilancia de los puentes no ha sufrido sensibles cambios de fondo a lo largo de los años hasta el día de hoy.

La primera mención a la vigilancia de los puentes ferroviarios es la que se recoge en el artículo 54 de la instrucción para la redacción de proyectos de tramos metálicos, publicado en la Gaceta de Madrid. Núm. 297 de 24 de octubre de 1925 [3]. Trascribiéndose literalmente a la del 1956 [4], donde debido a su brevedad lo incluimos íntegramente a continuación: entre paréntesis ( ) se recogen las inclusiones en el texto de la norma de 1956.

"Articulo 54 (45). Tramos en servicio y de construcción anterior a la vigencia de esta instrucción. Una vez que se establece el servicio normal de un tramo metálico e independientemente de la vigilancia y conservación de carácter ordinario que debe hacerse con toda atención, se realizarán revisiones periódicas de estas estructuras.

Los resultados de estas inspecciones se harán constar en documentos especiales para formar con ellas la historia del tramo.

Durante la vigilancia normal se corregirá inmediatamente todo deterioro o avería susceptible de aumento o que pudiera comprometer la seguridad de la obra.

Debe renovarse la pintura de las partes descubiertas, y si es posible de las ocultas, tan frecuentemente como sea necesario para preservarlas de la oxidación.

Se realizará una revisión cada dos años de todos los tramos metálicos, la que tendrá principalmente por objeto comprobar el estado de los remaches (roblones), pernos, (cordones de soldadura) etc., comprobándose que las piezas sometidas a esfuerzos de compresión no han experimentado deformación permanente; se golpearán todas la piezas y remaches (roblones y cordones de soldadura) con el martillo para asegurarse no tiene grietas ni que estos (aquellos) se encuentran flojos, igualmente se apreciará la posición de los aparatos de apoyo.

Cada diez años, además de la inspección que pudiera corresponderle, descrita en el párrafo anterior, se realizarán pruebas estáticas y dinámicas idénticas a las prescriptas en los artículos 42 al 53 (33 al 44) para los tramos metálicos nuevos, comprobándose los esfuerzos máximos a los que estén sometidas todas las piezas y las deformaciones y oscilaciones generales y locales de todos los elementos.

Cuantas prescripciones se detallan anteriormente se aplicarán a los tramos metálicos de construcción anterior a la fecha en que entre en vigor la Instrucción, y que igualmente han entrado en su servicio normal con prioridad a dicha fecha, admitiéndose coeficientes de trabajo y de deformación con tolerancias del 25 por 100 sobre los fijados para los tramos de nueva construcción." (este último párrafo se omite en la Instrucción del 56). [3] [4]

Estas instrucciones de 1925 y 1956 quedaron derogadas por la aprobación de la orden ministerial de 25 de junio de 1975, instrucción relativa a las acciones a considerar en el proyecto de puentes de ferrocarril [5]. En esta se modifica el texto, pero no hay un cambio conceptual técnico en cuanto a la vigilancia a realizar para garantizar la seguridad de las estructuras, continuando con la necesidad de realizar inspecciones visuales y pruebas de carga, aportando, con respecto a las anteriores, una mayor precisión del alcance de las mismas. En cuanto a las inspecciones visuales: "todos los puentes, cualquiera que sea su luz y material de que estén realizados" [5], y en cuanto a las pruebas de carga: "puentes metálicos de luz mayor de 12 metros" [5].

Hubo que esperar hasta 2005 para tener una instrucción dedicada expresamente a la vigilancia de las estructuras ferroviarias. Este importante documento recoge y desarrolla como no se había hecho antes, el objeto, el alcance y la consistencia de las inspecciones visuales, introduciendo los conceptos de inspección básica e inspección principal, así como la posibilidad desarrollar otros trabajos bajo el concepto de inspección especial.

Más importante si cabe, es que por primera vez regula la formalización administrativa de la vigilancia lo que permite una mejor supervisión y control de la actividad. Sin embargo, no hay cambios estructurales en cuanto a las técnicas a aplicar para garantizar la seguridad ferroviaria, basándose estas, como en todas las antecedentes, en la vigilancia visual y las pruebas de carga de los puentes metálicos.

\section{3. \\ DESARROLLO ACTUAL DE LAS NORMAS E INSTRUCCIONES}

Para avanzar en el desarrollo y evolución del mantenimiento de los puentes ferroviarios y a raíz del trabajo realizado durante las últimas décadas por los diferentes responsables del mantenimiento, con la experiencia adquirida en los miles de inspecciones y de los cientos de pruebas de carga realizadas, se comenzó en 2018 el proceso de actualización y mejora de los procesos internos y las normas de ADIF que complementan a la ITPF-05 [1]. Este proceso empieza a ver sus frutos en el año 2019 con la aprobación de los primeros documentos.

Con ello se pretende regular en detalle la manera en la que se deben realizar las actividades propias del mantenimiento y en especial las inspecciones y las pruebas de carga. 
Mientras que las normas describen en detalle cómo deben de realizarse técnicamente las actividades, las instrucciones técnicas recogen cómo deben articularse dichas actividades dentro de la estructura organizativa de Adif describiendo los procesos y las fases de trabajo que conlleva su realización, con el objetivo de contribuir a la consecución de los correspondientes indicadores de prestación de servicio de manera segura y eficiente durante la fase de mantenimiento del ciclo de vida de los activos ferroviarios. Estos nuevos planteamientos buscan dar respuesta a los siguientes aspectos:

- Evaluar de los procesos de mantenimiento, para detectar y analizar los riesgos operativos, organizativos y técnicos significativos. Establecer las funciones de las distintas áreas de la empresa involucradas en el mantenimiento y desarrollar un sistema para supervisar la eficacia de las medidas adoptadas.

- Planificar y controlar las operaciones de mantenimiento, recabando información para medir la correcta aplicación y eficacia de los procesos, garantizando que dichos procesos sean conformes a los requisitos de seguridad.

- Desde el punto de vista de la gestión de activos:

o Es fundamental realizar la gestión de riesgos asociados a los activos físicos a lo largo de todo su ciclo de vida, desde su diseño hasta su retirada.

o Garantizar también que los activos se utilicen para los fines previstos, que estén en condiciones de operar de manera segura y que mantengan el nivel de rendimiento previsto, tanto en condiciones normales como degradadas.

o Detectar los casos de incumplimientos de requisitos operativos y aplicación de restricciones de uso que sean convenientes. o Garantizar la conformidad de los procesos de gestión de activos con los requisitos clave de las especificaciones técnicas de interoperabilidad (ETI) [6] y otros requisitos pertinentes.

o En lo referente al control de la información y comunicación, se pretende el intercambio de la información dentro de la organización, para ello es fundamental garantizar la trazabilidad de toda la información, la elaboración y conservación de los registros.

- También se tiene en cuenta la relación con los contratistas, los socios y proveedores:

o Detectando y controlando los riesgos derivados de la necesaria externalización de algunas de las actividades de vigilancia y control de las estructuras.

o También, se supervisa la eficiencia en materia de seguridad de las actividades desempeñadas por los contratistas, así como su concienciación en dicha materia.

- Gestionar el cambio continuo mediante la aplicación y control de las modificaciones producidas en el sistema de mantenimiento.

- Supervisar los procesos de mantenimiento, verificando que se aplican correctamente y que se obtienen los resultados previstos, aplicando, en su caso, las medidas correctoras necesarias.

Es por ello que para cubrir todos estos objetivos se están desarrollado una serie de normas e instrucciones técnicas. A la fecha de redacción del presente artículo, la situación de estos documentos relativos al mantenimiento de los puentes ferroviarios es la siguiente:

TABLA 1.

Estado de desarrollo de los diferentes documentos relativos al mantenimiento de puentes ferroviarios en ADIF. (Fuente: Elaboración propia)

\begin{tabular}{|c|c|c|c|}
\hline ACTIVIDAD & RESULTADOS PRINCIPALES & $\begin{array}{l}\text { ACTIVIDAD QUE } \\
\text { DESENCADENA }\end{array}$ & $\begin{array}{l}\text { NORMA ADIF } \\
\text { PLATAFORMA }\end{array}$ \\
\hline $\begin{array}{l}\text { Inspección básica de puentes } \\
\text { de ferrocarril (IB). }\end{array}$ & $\begin{array}{l}\text { Detección precoz de potenciales } \\
\text { patologías estructurales de los puentes de } \\
\text { ferrocarril (potenciales daños N3 y N4). }\end{array}$ & $\begin{array}{l}\text { Inspecciones principales. Actuaciones } \\
\text { de mantenimiento no estructurales. }\end{array}$ & $\begin{array}{l}\text { Aprobada el 29/01/2020 } \\
\text { NAP 2-4-0.0_1E [7] }\end{array}$ \\
\hline $\begin{array}{l}\text { Inspección principal de } \\
\text { puentes de ferrocarril (IP). }\end{array}$ & $\begin{array}{l}\text { Valoración de los daños de los puentes } \\
\text { de ferrocarril (N1, N2, N3 o N4, } \\
\text { compatible con la ITPF-05). }\end{array}$ & $\begin{array}{l}\text { Gestión de los defectos N3 } \\
\text { y N4 (daños de PF). } \\
\text { Plan de puentes. }\end{array}$ & $\begin{array}{l}\text { Aprobada el } \\
22 / 07 / 2020 \\
\text { NAP 2-4-1.0 [8] }\end{array}$ \\
\hline $\begin{array}{l}\text { Prueba de carga de puentes } \\
\text { de ferrocarril (PC). }\end{array}$ & $\begin{array}{l}\text { Comprobación de la aptitud del puente } \\
\text { según ITPF-05 (apto, no apto). } \\
\text { Aptitud Adif (A0, A1, A2). } \\
\text { Categoría EN-15528 (D4-120, C3-350). } \\
\text { Clasificación ETI. } \\
\text { Capacidad ante transportes excepcionales. }\end{array}$ & $\begin{array}{l}\text { Gestión de la falta de } \\
\text { aptitud del puente. } \\
\text { Categorización de la línea (EN15528). } \\
\text { Viabilidad estructural ante } \\
\text { transportes excepcionales. }\end{array}$ & $\begin{array}{l}\text { Aprobada el 22/01/2021 } \\
\text { NAP 2-4-2.0 [9] }\end{array}$ \\
\hline $\begin{array}{l}\text { Inspección especial de } \\
\text { caracterización dinámica de } \\
\text { puentes de ferrocarril (IECD). }\end{array}$ & $\begin{array}{l}\text { Modelo analítico. } \\
\text { Parámetros que caracterizan } \\
\text { el comportamiento dinámico } \\
\text { del puentes de ferrocarril. } \\
\text { Detección precoz de } \\
\text { anomalías estructurales. }\end{array}$ & $\begin{array}{l}\text { Estudios de compatibilidad } \\
\text { dinámica del material móvil } \\
\text { de nuevas operadoras. }\end{array}$ & En redacción. \\
\hline $\begin{array}{l}\text { Inspección especial de cauce } \\
\text { de puentes de ferrocarril }\end{array}$ & $\begin{array}{l}\text { Valoración de capacidad hidráulica } \\
\text { y potencial erosivo del cauce. }\end{array}$ & Inspección subacuática. & En redacción. \\
\hline $\begin{array}{l}\text { Inspección especial subacuática } \\
\text { de puentes de ferrocarril. }\end{array}$ & $\begin{array}{l}\text { Valoración de la socavación y } \\
\text { estado del lecho fluvial. }\end{array}$ & $\begin{array}{l}\text { Valoración de la socavación y } \\
\text { estado del lecho fluvial. }\end{array}$ & En redacción. \\
\hline $\begin{array}{l}\text { Inspección principal de } \\
\text { pasos superiores. }\end{array}$ & $\begin{array}{l}\text { Valoración de los daños de los puentes } \\
\text { de ferrocarril (N1, N2, N3 o N4). }\end{array}$ & $\begin{array}{l}\text { Gestión de los defectos N3 } \\
\text { y N4 (daños del PS). }\end{array}$ & $\begin{array}{l}\text { Aprobada el 22/01/2021 } \\
\text { NAP 2-4-1.4 [10] }\end{array}$ \\
\hline
\end{tabular}


Se observa por tanto que ya están aprobadas las tres normas asociadas a las inspecciones básicas y principales de los puentes ferroviarios y la de inspecciones principales de pasos superiores. Estas normas constituyen una barrera preventiva de control del riesgo de degradación de los activos durante la totalidad de su ciclo de vida.

Para explicar el alcance de la nueva metodología nos centraremos en las inspecciones básicas que se desarrollan en la "Norma Adif Plataforma. Inspección básica de puentes de ferrocarril" (NAP 2-4-0.0) [7]. Esta norma consiste en describir la actividad de la inspección básica según los preceptos marcados por la "instrucción sobre las inspecciones técnicas en los puentes de ferrocarril" (ITPF-05) [1], es decir, la inspección visual realizada por personal de vigilancia e inspección general de la línea, de todos los elementos del puente para detectar y documentar la existencia de daños, asignando a cada daño detectado una determinada intensidad. Esta intensidad servirá para establecer el estado de avance o la extensión del daño detectado. La diferencia entre esta nueva metodología y la anterior estriba en que al inspector lo que se le pide es que identifique los daños y que determine el grado de evolución del daño que observa en función de los criterios recogidos en la norma, pero no que evalúe la estructura o que se manifieste sobre las consecuencias que ese daño puede tener en garantizar la capacidad estructural. De esta forma se consigue desvincular al inspector del resultado de la inspección básica de forma que se le confiere un carácter de observador de daños y no de evaluador de estructuras, lo que le permite realizar su función de manera más objetiva. No hay que perder de vista que el objetivo de las inspecciones básicas es "detectar lo antes posible, y sin esperar a la siguiente inspección principal programada, la aparición de cualquier daño real o aparente susceptible de seguimiento o reparación" ${ }^{\prime \prime}$, es decir, alertar de la necesidad de adelantar la realización de la próxima inspección principal sin agotar el plazo establecido según los criterios generales de su mantenimiento cíclico. Así pues, será el inspector que realice la inspección principal, "técnico titulado con competencia legal y con experiencia probada en patología estructural" , quien deba realizar una caracterización más detallada de los daños y el análisis y evaluación de estos.

Para estructurar y homogeneizar los resultados obtenidos y para facilitar la labor del inspector de las inspecciones básicas se han analizado todos los posibles daños que pueden ser detectados en la inspección visual. Se ha establecido dentro de la propia norma un catálogo de daños específico para la inspección básica, donde se distingue entre elementos estructurales (estribos, pilas y vanos) y no estructurales y entre los diferentes tipos de materiales (fábrica, metálico y hormigón armado). Estos daños se han analizado distinguiendo para cada uno 4 niveles de intensidad que reflejan 4 fases de la evolución del daño.

Para facilitar el trabajo del inspector el catálogo de daños de la norma dispone para cada daño de una ficha que recoge los aspectos más relevantes y los cuatro niveles de intensidad con una descripción de los mismos.

Los daños se han categorizado en función de la repercusión máxima, o nivel de gravedad máximo, que ese daño podría lle-

1 Apartado 3.1 Objeto, de la instrucción sobre las inspecciones técnicas en los puentes de ferrocarril (ITPF-05)

2 Apartado 2.4 Personal inspector, de la instrucción sobre las inspecciones técnicas en los puentes de ferrocarril (ITPF-05)

\section{Dadif}

ANEJO I CATÁLOGO DE DANNOS PARA INSPECCIÓN BÁSICA DE PUENTES DE FERROCARRIL

\begin{tabular}{|l|l|l|l|l|l|}
\hline \multicolumn{2}{|l|}{ NOMBRE DEL DAÑO: } & \multicolumn{2}{l|}{ SOcavación } & CODIGO DAÑO: & 3.F.S \\
\hline ELEMENTO: & Pila & MATERIAL: & FÁBRICA & CATEGORIA: & 4 \\
\hline DESCRIPCIÓN:
\end{tabular}

Daño que supone la pérdida de terreno sobre el que se apoya la cimentación, como consecuencia de la formacion de cavidades y a descompresión del terreno en el entorno de las bases de apoyo a causa de la acción de las corrientes de agua.
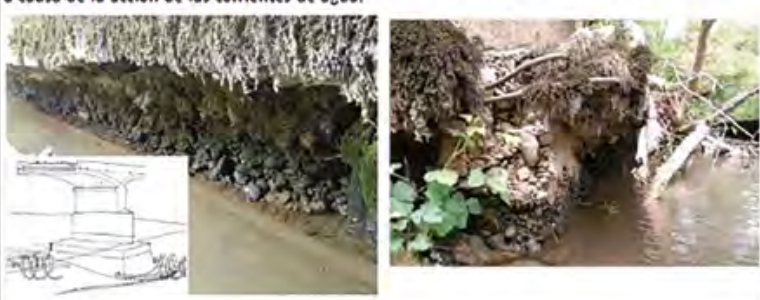

OBSERVACIONES:

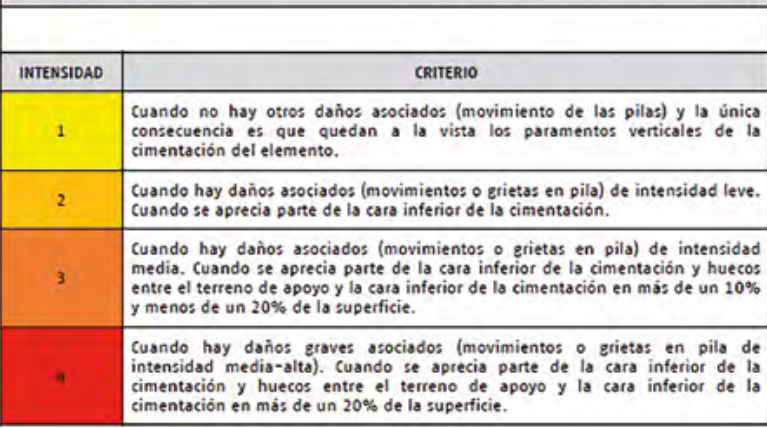

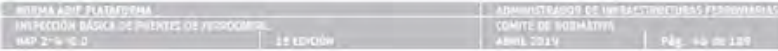

Figura 1. Ficha del daño "socavación", del elemento portante "pila" y material "fábrica". (Fuente "Norma Adif Plataforma. Inspección básica de puentes de ferrocarril" NAP 2-4-0.0) [7]

gar a tener sobre la seguridad estructural o de la explotación ferroviaria, de forma que al establecer la intensidad del daño se obtiene automáticamente un nivel de gravedad.

La categoría de un daño se define como el nivel de gravedad máximo que podría alcanzar ese defecto para un elemento concreto del activo en su estado más avanzado de evolución. La categoría del daño depende, por tanto, del tipo de daño y del elemento en el que se observa el daño.

\section{Categoría del daño $=f($ tipo de defecto, elemento afectado $)$}

\section{TABLA 2.}

Niveles de gravedad en función de la categoría del daño y de la intensidad en que se presenta. (Fuente "Norma Adif Plataforma. Inspección básica de puentes de ferrocarril" NAP 2-4-0.0) [7]

\begin{tabular}{|c|c|c|c|c|c|}
\hline & \multicolumn{4}{|c|}{ INTENSIDAD DEL DAÑO } \\
\hline & & Intensidad 1 & Intensidad 2 & Intensidad 3 & Intensidad 4 \\
\hline \multirow{4}{*}{ 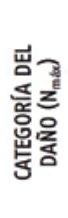 } & Categoría 1 & N1 & N1 & N1 & N1 \\
\hline & Categoría 2 & N1 & N2 & N2 & N2 \\
\hline & Categoría 3 & N1 & N2 & N3 & N3 \\
\hline & Categoría 4 & N1 & N2 & N3 & N4 \\
\hline
\end{tabular}


Así, podemos decir que el nivel de gravedad de un determinado daño que se aprecia en un puente es función del tipo de defecto, del elemento afectado y de la intensidad en que se presenten el daño.

\section{Nivel de gravedad de un daño (NGD) = $f($ tipo de defecto, elemento afectado, intensidad)}

Los niveles de gravedad del daño se interpretan según la siguiente tabla.

TABLA 3.

Escala y descripción de los niveles de gravedad de daños. (Fuente "Norma Adif Plataforma. Inspección básica de puentes de ferrocarril" NAP 2-4-0.0) [7]

\begin{tabular}{|c|l|}
\hline $\begin{array}{c}\text { NIVEL DE GRAVEDAD } \\
\text { DEL DAN0 }\end{array}$ & \multicolumn{1}{|c|}{ DESCRIPCION } \\
\hline N1 & $\begin{array}{l}\text { Defectos sin repercusión en el comportamiento estructural del activo, } \\
\text { ni en la explotación ferroviaria, ni en Ia durabilidad o funcionalidad } \\
\text { del elemento. }\end{array}$ \\
\hline N2 & $\begin{array}{l}\text { Defectos sin repercusión en el comportamiento estructural del activo, } \\
\text { ni en la explotación ferroviaria, pero que menoscaban la durabilidad } \\
\text { o la funcionalidad del elemento. }\end{array}$ \\
\hline N3 & $\begin{array}{l}\text { Defectos que evidencian una evolución patológica y pueden afectar a } \\
\text { la seguridad estructural o a la explotación ferroviaria, o que afectan a } \\
\text { terceros. }\end{array}$ \\
\hline N4 & $\begin{array}{l}\text { Defectos que afectan a la seguridad estructural o a la explotación } \\
\text { ferroviaria. }\end{array}$ \\
\hline
\end{tabular}

Según el planteamiento realizado habrá algunos defectos que, aunque se presenten en su nivel más alto de intensidad solo lleguen a provocar niveles intermedios o bajos de gravedad; como por ejemplo el daño: "Pintadas/Grafitis", por mucho que aumente su intensidad, es decir, aunque esté presente en toda la estructura, su nivel de gravedad siempre será $\mathrm{Nl}$, luego la categoría de este daño es 1 . La "presencia de vegetación enraizada herbácea, musgos o líquenes", si se presenta en su mayor nivel de intensidad alcanza un nivel de gravedad 2, luego se trata de un daño de categoría 2. Pero existirán daños que en su nivel más alto de intensidad provocarán un nivel de gravedad máximo como por ejemplo el "descalce o la socavación de las pilas" que presenta para la intensidad 4 un nivel de gravedad 4; por tanto, la categoría de este daño es 4 .

En la propia norma se incluye como anejo las matrices de nivel de gravedad en las inspecciones básicas de puentes de ferrocarril.

Es interesante destacar que algunos defectos, a medida que aumentan su intensidad, pueden desencadenar la aparición de otras patologías. Un ejemplo podría ser cómo el daño de "vegetación enraizada herbácea" puede dar origen al daño "vegetación enraizada arbórea" y esta a su vez al daño "Deformaciones / Abombamientos / Desplome". Es por ello por lo que es preciso mantener una vigilancia exhaustiva y mantenida en el tiempo de las estructuras.

En total se han establecido para las inspecciones básicas 150 tipos de defectos distribuidos en 21 subcategorías.

Como ya se ha indicado, el inspector no tiene que determinar el nivel de gravedad de un daño, este se obtendrá automáticamente a través de una aplicación informática desarrollada al efecto que procesa estas matrices. Se determina por tanto el

\section{ANEJO 2. MATRICES DE NIVEL DE GRAVEDAD EN INSPECCIONES bÁSICAS DE PUENTES DE FERROCARRIL}

Las matrices de nivel de gravedad en función del tipo de daño, el elemento afectado (en algunos casos diferenciando por material de construcción) y la intensidad del dano son las siguientes:

\begin{tabular}{|c|c|c|c|c|c|}
\hline \multirow{2}{*}{$\begin{array}{c}\text { TABLA: NIVEL DE GRAVEDAD del catálogo de daños } \\
\text { DANNO }\end{array}$} & \multirow[b]{2}{*}{ Nmax } & \multicolumn{4}{|c|}{$\begin{array}{l}\text { INTENSIDAD DEL } \\
\text { DANO }\end{array}$} \\
\hline & & 1 & 2 & 3 & 4 \\
\hline \multicolumn{6}{|l|}{ 1. TERRENO CIRCUNDANTE } \\
\hline \multicolumn{6}{|l|}{ 1.1. Terraplenes de acceso } \\
\hline Hundimiento / Deslizamiento & 4 & N1 & $\mathrm{N2}$ & N3 & N4 \\
\hline Erosión / Cárcavas & 4 & N1 & $\mathrm{N2}$ & N3 & N4 \\
\hline Rotura / Fisura / Pérdida de piezas / Deterioro del encachado & 2 & N1 & 112 & $\mathrm{N2}$ & $\mathrm{N} 2$ \\
\hline \multicolumn{6}{|l|}{ 1.2. Cauce } \\
\hline Descalce / Socavación & 4 & N1 & 112 & N3 & N4 \\
\hline Rotura / Deterioro de protecciones & 2 & N1 & $\mathrm{N2}$ & $\mathrm{N} 2$ & $\mathrm{~N} 2$ \\
\hline Erosión de las márgenes & 2 & N1 & N2 & $\mathrm{N2}$ & N2 \\
\hline Aterramiento del vano & 3 & N1 & N2 & N3 & N3 \\
\hline \multicolumn{6}{|l|}{ 2. ESTRIBO } \\
\hline \multicolumn{6}{|l|}{ Estribo fábrica (incluye hormizón en masa) } \\
\hline Fisuras / Grietas & 4 & N1 & $\mathrm{N2}$ & N3 & N4 \\
\hline $\begin{array}{l}\text { Grietas de asentamiento diferencial estribo y Muro de } \\
\text { acompanamiento-Aletas }\end{array}$ & 2 & N1 & N2 & N2 & N2 \\
\hline Descuelque, desconfizuración del aparejo o perdida de piezas & 4 & N1 & $N 2$ & N3 & N4 \\
\hline Deformaciones / Abombamientos / Desplome & 4 & N1 & 112 & N3 & N4 \\
\hline Asientos / Movimientos / Giros & 4 & N1 & $\$ 2$ & N3 & N4 \\
\hline Descalce / Socavación & 4 & N1 & 112 & N3 & N4 \\
\hline Humedades, pátinas, costras o eflorescencias & 2 & N1 & $\mathrm{N} 2$ & $\mathrm{N2}$ & $\mathrm{N2}$ \\
\hline Vegetación enraizada arbórea & 3 & N1 & N2 & N3 & N3 \\
\hline Vegetación enraizada herbácea, musgos o líquenes & 2 & N1 & $\mathrm{N2}$ & $\mathrm{N} 2$ & $\mathrm{~N} 2$ \\
\hline Degradación superficial & 3 & N1 & $\mathrm{N2}$ & N3 & N3 \\
\hline
\end{tabular}

Figura 2. Ejemplo de matriz de nivel de gravedad para terraplenes de acceso, cauce y estribo de hormigón en masa. (Fuente "Norma Adif Plataforma. Inspección básica de puentes de ferrocarril” NAP 2-4-0.0) [7] 
TABLA 4.

Extracto de la tabla general incluida en el documento "Criterios generales de mantenimiento preventivo de la especialidad de infraestructura y vía" [1] para el caso de la inspección básica de puentes.

\begin{tabular}{|c|c|c|c|}
\hline $\begin{array}{l}\text { ORIGEN DE LA } \\
\text { ACIIVIDAD SEGON } \\
\text { ESTADO }\end{array}$ & CONSISIENCAA SEGON ETADO & ACIUACION & EN CASO DE INCUMPUMIENTO \\
\hline \multirow{3}{*}{$\begin{array}{l}\text { Por evolución de } \\
\text { un daño detectado } \\
\text { en una IB }\end{array}$} & $\begin{array}{c}\text { Si algún defecto de } \\
\text { categoría } 3 \text { asciende a N3, } \\
\text { verificado por la Jefatura de } \\
\text { Puentes y según la } \\
\text { instrucción IT-301-001-30 } \\
\text { y la norma NAP 2-4-0.0 } \\
\text { Inspección básica de } \\
\text { puentes de ferrocarril }\end{array}$ & $\begin{array}{l}\text { IP en un plazo inferior a } 2 \\
\text { años tras la fecha de } \\
\text { ejecución de la IB, si no } \\
\text { está ya programada en un } \\
\text { plazo inferior }\end{array}$ & $\begin{array}{c}\text { Reanálisis de las IB por parte de } \\
\text { la Jefatura de Puentes } \\
\text { confirmando que no existe } \\
\text { evolución. }\end{array}$ \\
\hline & $\begin{array}{c}\text { Si algún defecto de } \\
\text { categoría } 4 \text { asciende a N3, } \\
\text { verificado por la Jefatura de } \\
\text { Puentes, según la } \\
\text { instrucción IT-301-001-30 } \\
\text { y la norma NAP 2-4-0.0 } \\
\text { Inspección básica de } \\
\text { puentes de ferrocarril }\end{array}$ & $\begin{array}{l}\text { Realizar una IP en un plazo } \\
\text { inferior a } 1 \text { año tras la } \\
\text { fecha de ejecución de la IB, } \\
\text { sĩ no está ya programada } \\
\text { en un plazo inferior }\end{array}$ & $\begin{array}{l}\text { Reanálisis de las IB por parte de } \\
\text { la Jefatura de Puentes } \\
\text { confirmando que no existe } \\
\text { evolución, y visita de un técnico } \\
\text { experto que confirme in situ la } \\
\text { evolución del daño en concreto. }\end{array}$ \\
\hline & $\begin{array}{l}\text { Si algún defecto alcanza el } \\
\text { N4, verificado por la } \\
\text { Jefatura de Puentes, según } \\
\text { la instrucción IT-301-001- } \\
30 \text { y la norma NAP 2-4-0.0 } \\
\text { Inspección básica de } \\
\text { puentes de ferrocarril }\end{array}$ & $\begin{array}{l}\text { IP en un plazo inferior a } 3 \\
\text { meses tras la fecha de } \\
\text { ejecución de la IB, si no } \\
\text { está ya programada en un } \\
\text { plazo inferior. } \\
\text { Implantación de } \\
\text { restricciones por parte de la } \\
\text { Jefatura o Jefatura de Área } \\
\text { de Mantenimiento. }\end{array}$ & $\begin{array}{c}\text { Visita de un técnico experto que } \\
\text { confirme in situ la gravedad del } \\
\text { daño y las restricciones } \\
\text { implantadas. }\end{array}$ \\
\hline
\end{tabular}

"Índice de estado de un elemento según IB" que resulta ser el nivel de gravedad más alto de los defectos de un determinado elemento según la valoración de la inspección básica. Por último, se obtiene el "indice de estado del activo según IB" que es el nivel de gravedad más alto de los daños detectados en el activo durante la realización de la inspección básica.

El resultado de la inspección básica no solo dependerá del índice de estado del activo, sino que también dependerá de la evolución de los niveles de gravedad de los defectos detectados respecto a la anterior inspección básica, según los criterios establecidos en el documento "Criterios generales de mantenimiento preventivo de la especialidad de infraestructura y vía".

Como consecuencia de esta valoración se propone una determinada actuación que generalmente suele ser la realización de una inspección principal en un plazo determinado, una vez que haya sido verificada la inspección por la Jefatura de Puentes, aunque en los casos más extremos puede desembocar directamente en la implantación de restricciones al tráfico como medida más inmediata.

Con el desarrollo de estos nuevos documentos que regulan las inspecciones se consigue una homogeneidad en la evaluación y análisis de las estructuras tanto desde las inspecciones básicas como desde las inspecciones principales.

Por otra parte se están desarrollando los documentos para establecer los requerimientos mínimos que se deben exigir en el diseño y ejecución de las pruebas de carga que se exigen a los puentes ferroviarios, en cumplimiento de la ITPF-05 [1]. Estos requerimientos son de aplicación tanto en obra nueva, con el doble objetivo de verificar la adecuada concepción y ejecución y caracterizar adecuadamente el puente de forma que facilite su óptima explotación, como en los puentes en servicio, de cara a ampliar el conocimiento de su estado estructural.

Los criterios se establecen nuevamente en el documento "Criterios generales de mantenimiento preventivo de la especialidad de infraestructura y vía" [11] dependiendo de los ma- teriales y de la luz máxima de los vanos de la estructura.

Se está trabajando también en la redacción de documentos normativos para unificar las inspecciones de cauce, utilizando las metodologías desarrolladas para este tipo de inspecciones en años anteriores.

Aún así, cabe señalar que el desarrollo y la mejora constante del mantenimiento de los puentes no solo se fundamenta en organizar y homogeneizar las inspecciones de las estructuras en sus diferentes facetas (inspecciones visuales, pruebas de carga, inspecciones de cauce, ...), sino que también hay que destacar el empleo de nuevas tecnologías y desarrollos puestos a disposición del control y gestión de las estructuras.

\section{4.}

\section{NUEVOS SISTEMAS IMPLEMENTADOS PARA EL CONTROL Y MANTENIMIENTO}

Si bien la metodología de vigilancia basada en las inspecciones visuales ha sido suficiente a lo largo de estos 100 últimos años, parece que ha llegado el momento en el que convendría actualizarla. No decimos esto únicamente por el tiempo pasado, sino especialmente por los cambios acaecidos en los últimos 30 años. Hay dos aspectos fundamentales que han cambiado sustancialmente: por un lado, el significativo cambio en las características de los puentes que son objeto de la vigilancia, y por otro los nuevos medios y las técnicas de vigilancia desarrollados en los últimos años.

Como evidencia del cambio de las características de los puentes cabe señalar que la longitud media de las estructuras se ha duplicado pasando de los 50 a los 100 metros. Además antiguamente el porcentaje de los puentes con luz máxima superior a 25 metros era del $25 \%$, en los más modernos esta proporción asciende al 55\%. Pero tan importante como el signifi- 
cativo "crecimiento" de los puentes es el cambio en la filosofía del diseño de los mismos. En los antiguos podemos decir que se imponía una filosofía más ferroviaria en su diseño, en la que se buscaba minimizar la afección de la presencia del puente a la vía, recurriendo por lo general a puentes isostáticos en la que la compatibilidad de movimientos superestructura-estructura se va repartiendo en cada uno de los vanos. En los puentes construidos en los últimos 30 años se ha impuesto una filosofía estructuralista en su diseño, en la que buscando un ahorro en la construcción de los grandes viaductos, se ha recurrido con frecuencia a la ejecución de largas vigas continuas hiperestáticas, en las que la compatibilidad de movimiento estructura-superestructura se concentra en los extremos de estas vigas continuas. Queda claro, por tanto, que los nuevos puentes a mantener son, no solo más grandes, sino más complejos, en especial en la compatibilidad vía - estructura.

TABLA 5.

Comparativa de las luces máximas de vano entre los puentes anteriores y los posteriores al año 1990. (Fuente: Elaboración propia)

\begin{tabular}{lccc}
\hline Porcentaje de puentes de ffcc & \multicolumn{3}{c}{ Luz máxima de vano (m) } \\
& {$[10$ a 25] } & {$[\mathbf{2 5}$ a 50] } & $>\mathbf{5 0}$ \\
\hline Anterior a los 90 & $75 \%$ & $22 \%$ & $3 \%$ \\
Posteriores a los 90 & $45 \%$ & $44 \%$ & $11 \%$ \\
\hline
\end{tabular}

En cuanto a los medios y técnicas de vigilancia se ha producido, y se sigue produciendo, un enorme avance tecnológico.

Este avance tecnológico de los instrumentos de auscultación y de las comunicaciones ha ido aparejado a un descenso de sus costes de adquisición y explotación, lo que hace viable su uso común en la vigilancia de estructuras, e incluso bajo ciertas circunstancias (estructuras no accesibles, etc.) mucho más económicas que las inspecciones visuales. Este aspecto resulta fundamental y deberá tenerse en cuenta en la redacción de futuras instrucciones de manera que se permita que la monitorización de las estructuras supla las inspecciones visuales.

Aún más revolucionario que el avance descrito en la instrumentación será el uso de nuevas técnicas de vigilancia basadas en el análisis de la información recogida, lo que permitirá poder conocer, con anterioridad a que se revelen visualmente, anomalías o deficiencias del estado estructural del puente. Esta mejora redundará en un aumento de la fiabilidad de la estructura y en un mantenimiento más eficiente.

De forma similar a cómo en medicina se hacen campañas específicas de vigilancia de la salud a sectores particulares de la población, en los puentes se deberán hacer planes de vigilancia adaptados a sus particularidades o a las de sus elementos; decimos en estos casos que presentan un programa de mantenimiento ad hoc. Así, algunas situaciones para las que se debería realizar una vigilancia especial, distinta o complementaria a las inspecciones visuales, son:

- Estructuras o elementos estructurales con gran dificultad de acceso.

- Elementos de alta singularidad o responsabilidad estructural:

o Apoyos, aparatos de dilatación de grandes viaductos.

o Pilas con cimentaciones sensibles a los efectos de la socavación.

o Tirantes.

- Estructuras singulares o factores críticos que pudiesen afectar a la capacidad estructural.

- Evolución de la capacidad anticorrosión.

- Estructuras con actuaciones o reparaciones singulares cuya evolución deba ser controlada.

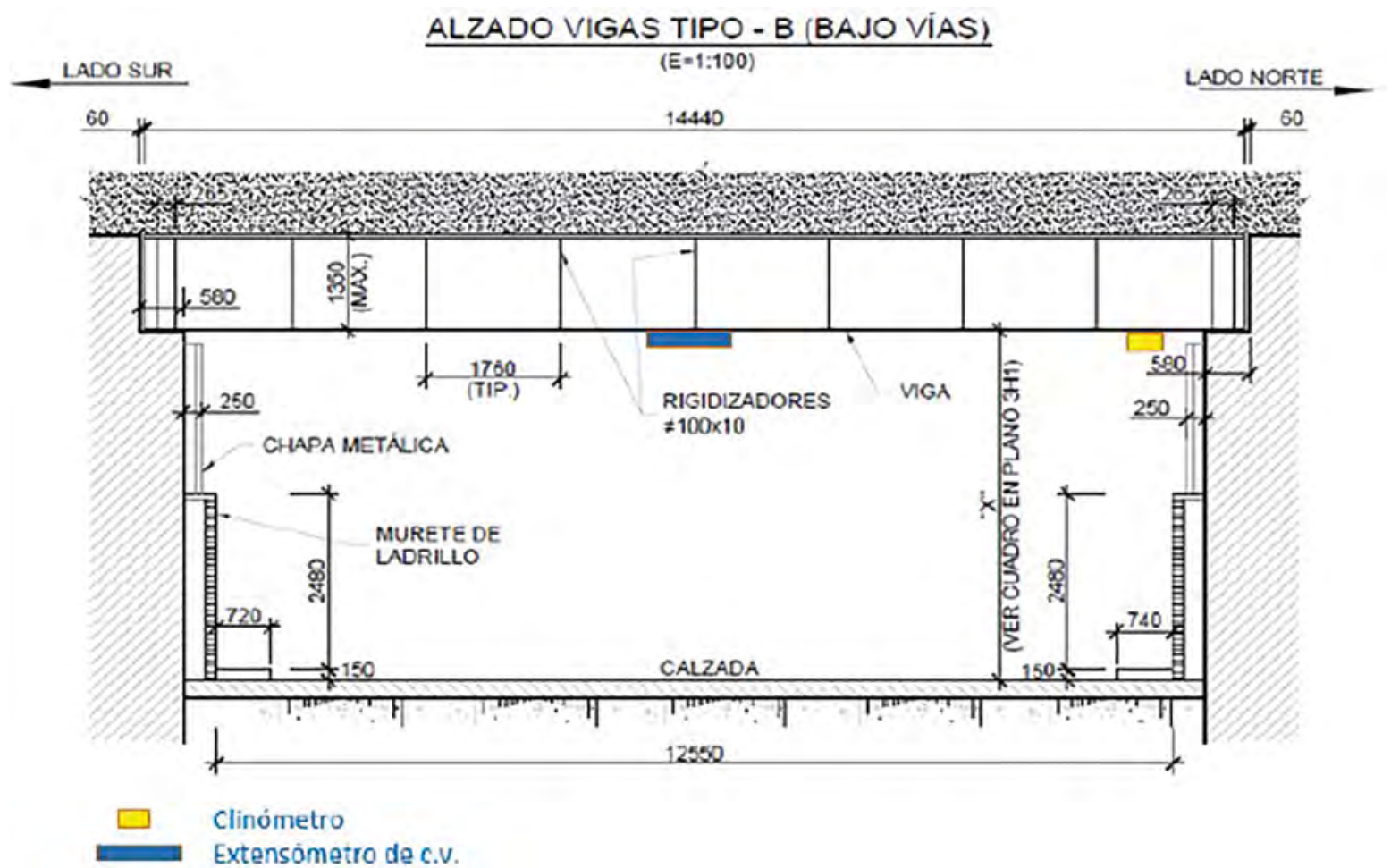

Figura 3. Ubicación de clinómetros y extensómetros de cuerda vibrante en estructura tipo de cubrición de calle bajo vías. (Fuente: Adif) 


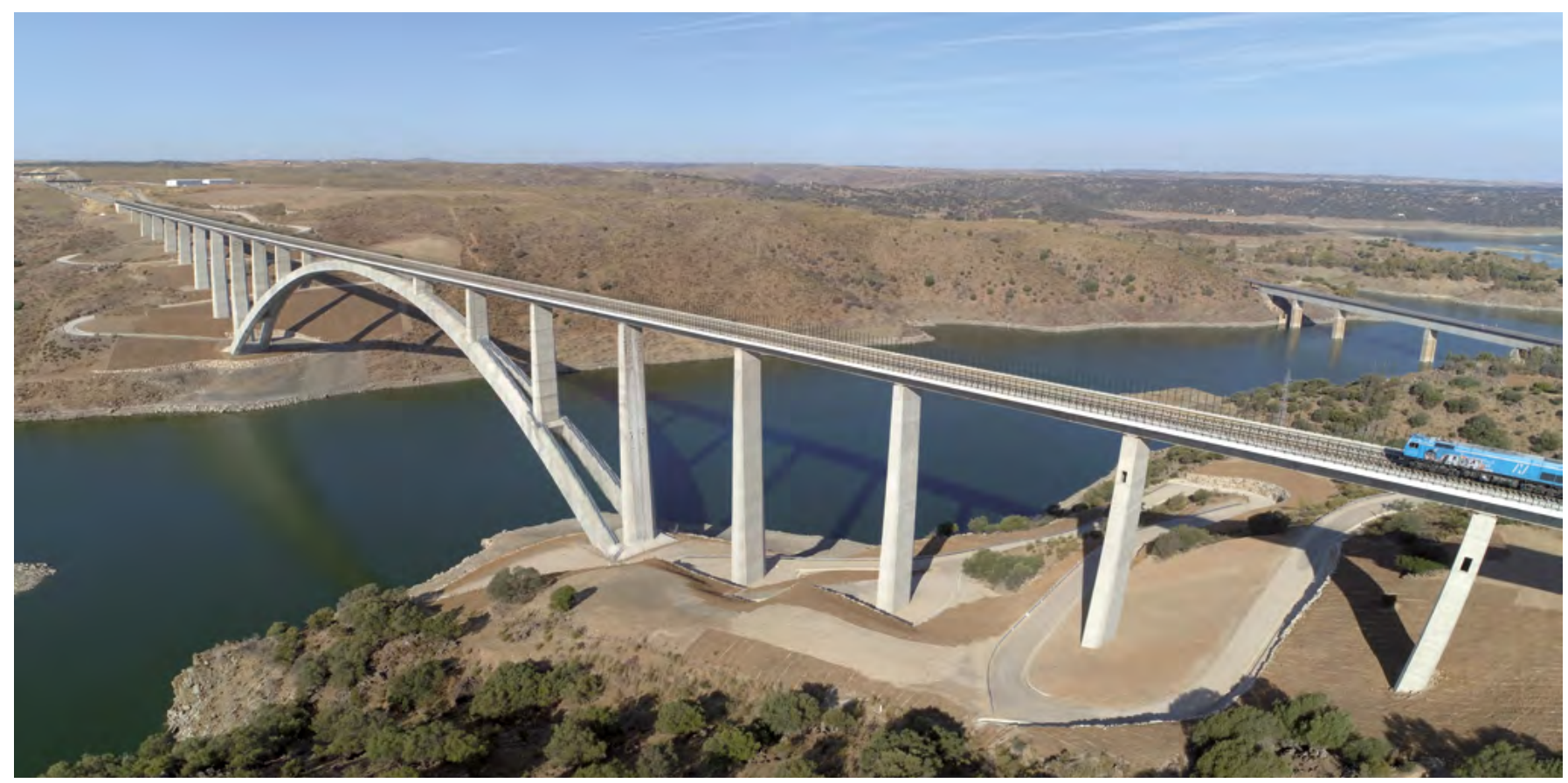

Figura 4. Vista general del viaducto de Almonte. (Fuente: Adif).

Existen determinadas estructuras que debido a su ubicación y funcionalidad presentan una grandísima dificultad para ser inspeccionadas de forma visual con la periodicidad requerida por las instrucciones técnicas y los criterios generales de mantenimiento. Es por ello por lo que deben disponerse otro tipo de sistemas para controlar las estructuras; un claro ejemplo serían las estructuras enterradas salvando otras infraestructuras en las que los sistemas de protección frente al fuego dificultan la inspección visual. En estas situaciones se hace recomendable, o incluso necesario, el uso de otros sistemas de vigilancia que nos faciliten información del estado estructural sin necesidad de descubrirlas debido a los elevadísimos costes económicos y de afección a los servicios públicos que esto conlleva.

Un ejemplo, de sistema equivalente a la vigilancia visual de este tipo de estructuras podría ser la disposición de extensómetros de cuerda vibrante y clinómetros para controlar el comportamiento de las mismas; deformaciones y giros. Esta información se transmite a la estación de adquisición de datos para su análisis posterior.

El control y vigilancia de estructuras singulares, así como de factores críticos que pudiesen afectar al comportamiento estructural, requieren de un seguimiento específico o incluso monitorización en remoto para conocer la evolución de su comportamiento. Una herramienta que sin duda en el futuro será de uso habitual es la del análisis modal del puente, cuyo objetivo es determinar los parámetros modales: las frecuencias naturales, los modos de vibración y las tasas de amortiguamiento asociados. La obtención de estos parámetros se realiza mediante el uso de técnicas de análisis de señal. Gracias al estudio de la evolución de estos parámetros podremos conocer el estado general del puente, así como identificar, con anterioridad a que sean visibles, daños que puedan afectar a la capacidad estructural.

Los métodos basados en el análisis modal se clasifican por el tipo de excitación que se utiliza en el ensayo. Si el ensayo se realiza con excitaciones artificiales controladas se denomina análisis modal experimental (EMA por sus siglas en inglés) y si el ensayo se realiza bajo excitaciones naturales se denomina análisis modal operacional (OMA por sus siglas en inglés), existe un tercer método disponible basado en la combinación de ambos, el Análisis Modal Operacional con entradas exógenas (OMAX).

Este es el caso, entre otros, del viaducto de Almonte, ubicado en la línea de alta velocidad Madrid-Extremadura-frontera portuguesa. Durante el proceso de construcción del viaducto se monitorizó la estructura a través de una instrumentación con medición continua que incluía clinómetros, acelerómetros, galgas extensométricas, células de carga, medidas topográficas automatizadas y medidas meteorológicas de viento y temperatura. El registro de todos estos valores permitía detectar desplazamientos o cambios en las frecuencias de vibración de la estructura que alertaría de cualquier cambio o anomalía en el comportamiento de la misma.

En la actualidad, a partir de esta instrumentación ya instalada, se va a dotar al viaducto de un sistema de monitorización en remoto; con ello se podrá evaluar su comportamiento evolutivo y cumplir los criterios de funcionalidad, seguridad y confort de los usuarios durante su vida útil.

La supervisión de la estructura llevada a cabo dentro del contrato de "Asistencia para el control de las obras" permitió durante los años de construcción, perfeccionar y calibrar los modelos de cálculo avanzado con los datos reales registrados, hasta obtener un "gemelo digital" de la estructura. Este gemelo digital es altamente sofisticado, pues responde al comportamiento real del viaducto enlazándolo de forma biunívoca con la monitorización. Todo ello permitirá en fase de explotación detectar con antelación suficiente las posibles patologías que puedan aparecer, resultando un mantenimiento preventivo más eficiente que el realizado a través de inspecciones convencionales. 
Durante todo este tiempo, el control de los parámetros reales ha permitido verificar el comportamiento sucesivo de la estructura. Incluso con eventos acaecidos de carácter extraordinario, se ha podido contrastar el correcto comportamiento del viaducto con respecto al comportamiento teórico obtenido del gemelo digital. Una vinculación que se ha demostrado esencial.

De igual forma, otros puentes, debido a los procesos sufridos durante su ejecución o durante la explotación de los mismos, requieren de sistemas de auscultación geotécnica y estructural que controle movimientos, giros y extensometría en los diferentes elementos constitutivos. Este es el caso del viaducto de "Pont Candí" perteneciente a la línea de alta velocidad Madrid-Zaragoza-Barcelona-Frontera francesa.

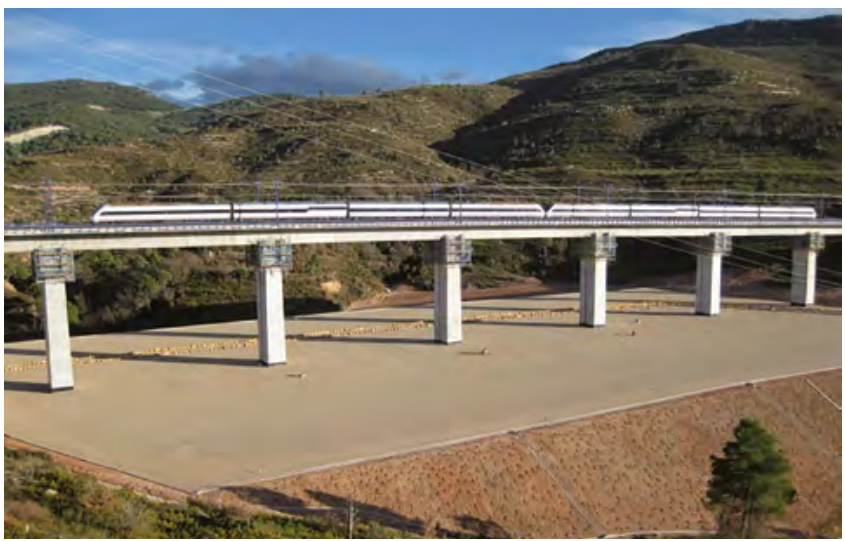

Figura 5. Vista general del viaducto de Pont Candi (Fuente: Adif).

En este caso se instalaron cerca de 400 dispositivos con el fin de controlar el terreno de cimentación, el cuerpo del relleno, el drenaje y las estructuras de bloqueo de pilas ejecutadas con posterioridad a la construcción. Además, se dispuso de una amplia red de nivelación que ha servido para controlar el terreno circundante y la propia estructura.

Otro aspecto que siempre ha preocupado a los gestores de infraestructuras es la detección precoz de los fenómenos de socavación. Este tipo de fenómenos en muchas ocasiones no son detectables a simple vista y requieren de la realización de inspecciones subacuáticas cuando la inspección del cauce así lo aconseja. En este sentido, Adif, está colaborando actualmente con la empresa constructora Tecsa y la Universidad Politécnica de Valencia (UPV) para el desarrollo de un sistema de monitorización de detección de la socavación en época temprana.

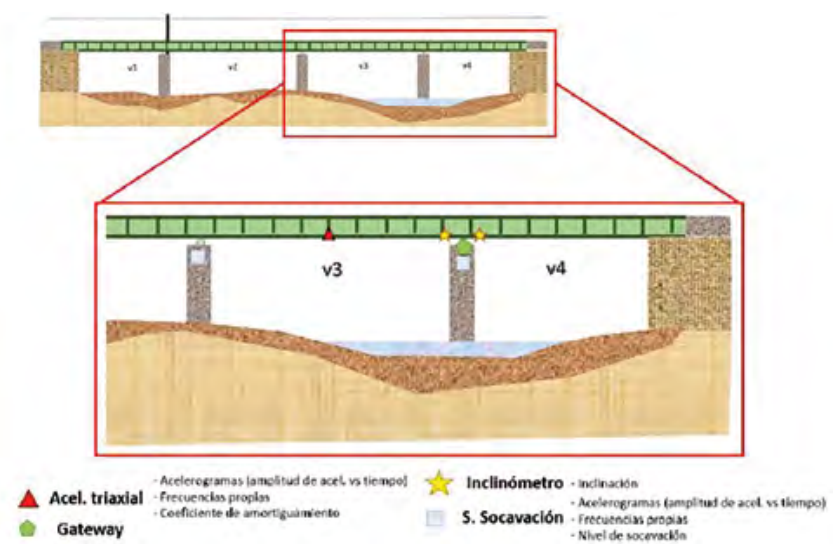

Figura 6. Esquema de distribución de equipos. (Fuente: Tecsa-UPV).

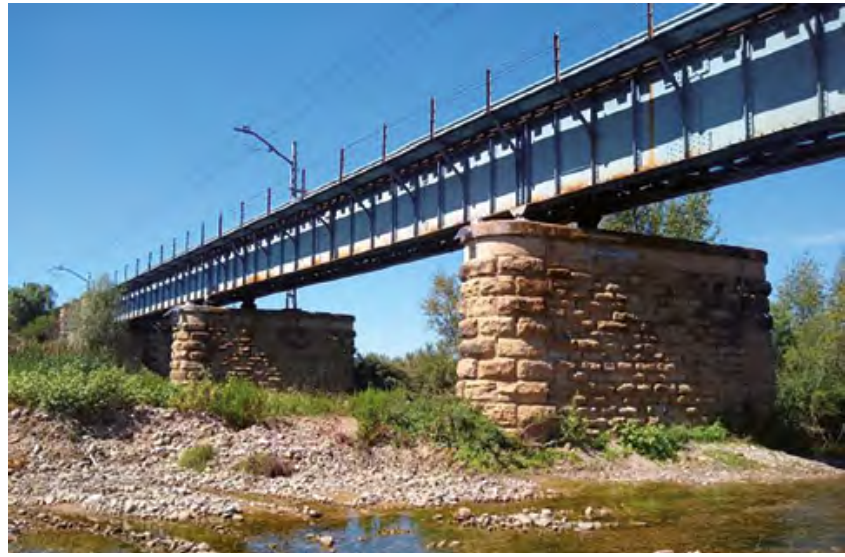

Figura 7. Vista general de equipos instalados en pilas del tramo metálico sobre el río Leza PK 065/097 línea Castejón-Bilbao. (Fuente: Tecsa-UPV).

Otro avance técnico que destacar en la realización de las inspecciones principales es el empleo de los drones como medio auxiliar. En los últimos años se ha empezado a utilizar los drones para trabajos civiles, y en el caso de las estructuras se han consolidado como un medio fundamental para realizar levantamientos geométricos, obtener modelos 3D de alta resolución para su uso en la redacción de proyectos o como herramienta sustitutiva de los medios auxiliares necesarios para llegar a todos los elementos del puente durante su inspección principal.

Desde el año 2019 ADIF está participando, junto con otras 10 administraciones ferroviarias europeas, en el proyecto DRONE4RAIL de la UIC (Union Internationale des Chemins de Fer) con objeto de definir una metodología y unos criterios para optimizar las inspecciones no intrusivas de los elementos de la infraestructura realizadas con drones. Este proyecto está alineado con la Rail Technical Strategy Europe (RTSE) [12], y actualmente se está redactando el documento que definirá los protocolos comunes y servirá de guía para los administradores ferroviarios.

El empleo de los drones ya es una realidad tanto para las inspecciones principales como para la redacción de proyectos debido a su versatilidad y rapidez. Uno de los ejemplos más recientes lo encontramos en el proyecto de rehabilitación del viaducto de "Martín Gil", situado en el PK 23/043 de la línea Zamora - A Coruña.

A la hora de afrontar la rehabilitación de este puente tan singular y emblemático, que fue récord del mundo de arco de hormigón en el momento de su construcción, se ha planteado la utilización de los drones como apoyo para poder realizar una definición completa del estado de esta estructura.

Se ha recurrido a esta herramienta que no solo permite acercar el ojo del inspector al puente, sin necesidad de otro tipo de estructuras auxiliares más costosas para poder evaluar los daños y patologías que presenta, sino que además permite realizar un levantamiento detallado de la estructura que resulta fundamental para poder definir con precisión las unidades de obra del proyecto de mejora.

El futuro y el rápido desarrollo de la tecnología aplicada a los drones no nos deja de sorprender; por ejemplo, recientemente se han realizado pruebas para inspección en remoto en tiempo real de puentes ferroviarios utilizando la tecnología 5G para comprobar la capacidad de transmisión de la información. 


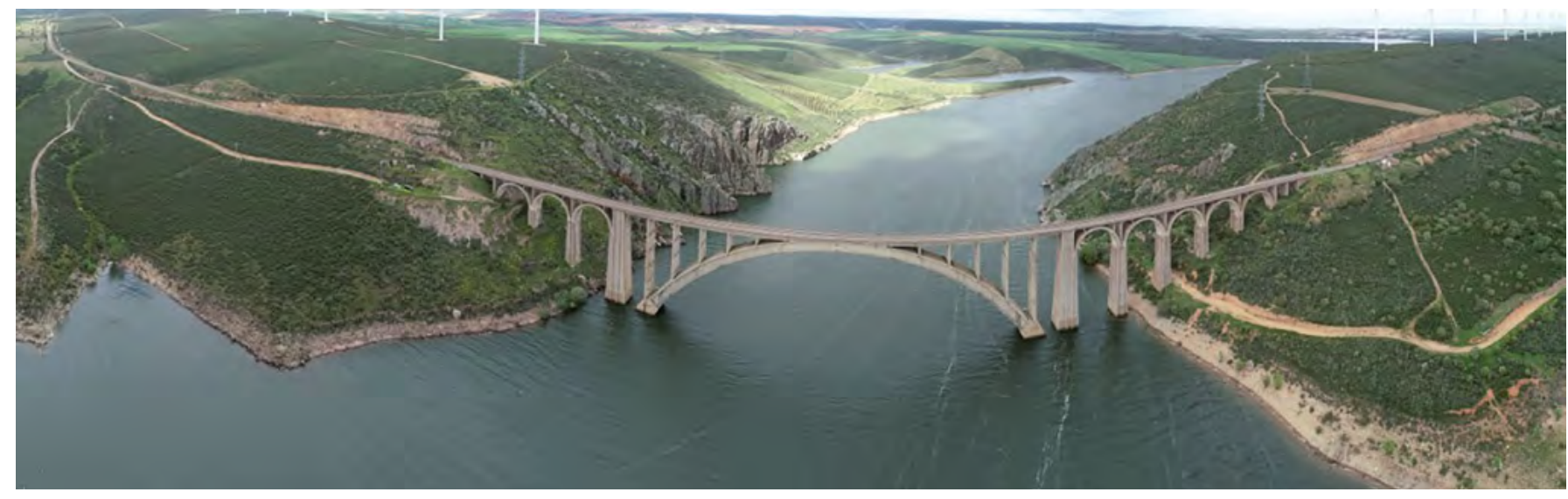

Figura 8. Vista general desde el dron del viaducto Martín Gil situado en el PK 23/043 de la Línea Zamora - A Coruña. (Fuente: Ineco).

转

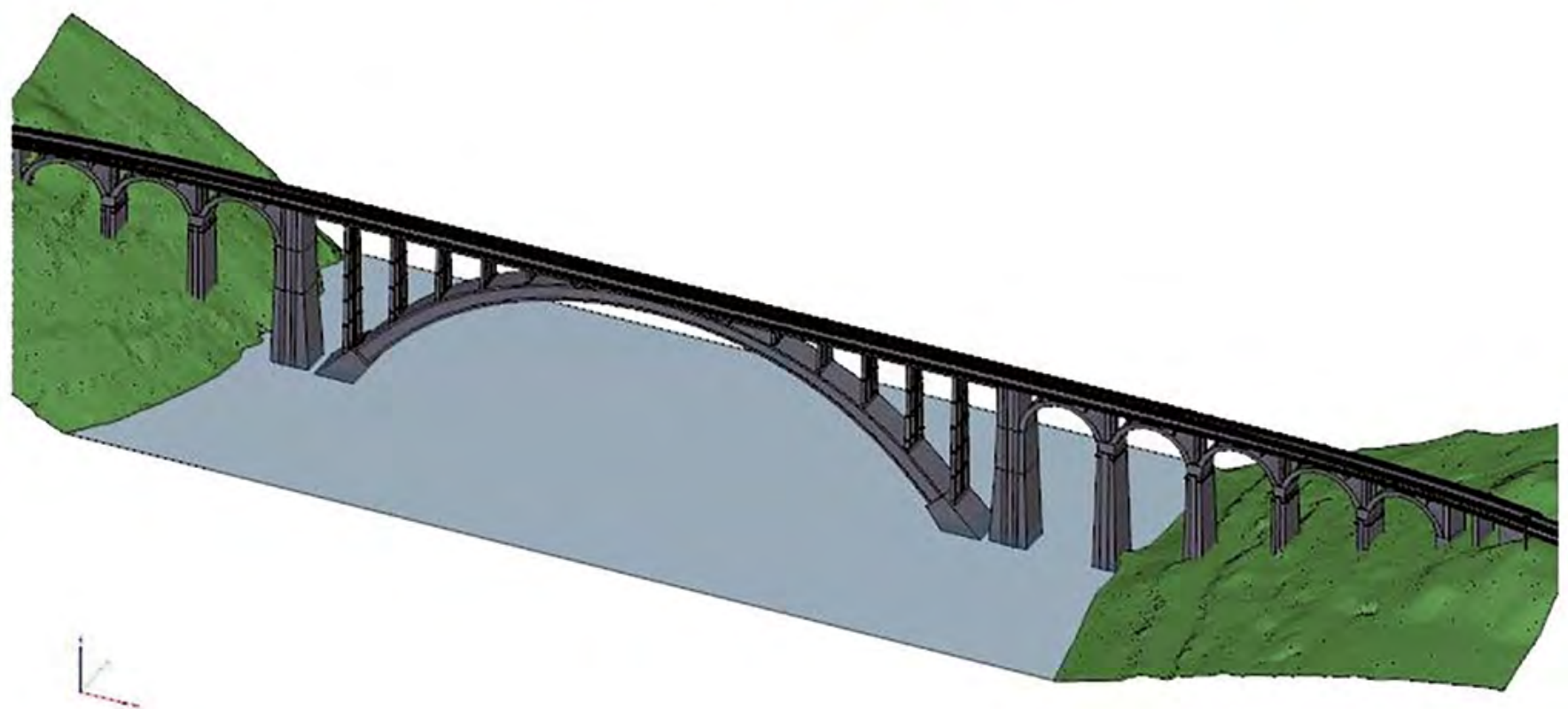

Figura 9. Modelo digital obtenido a través de los datos tomados con el vuelo del dron del viaducto Martín Gil. (Fuente: Ineco).
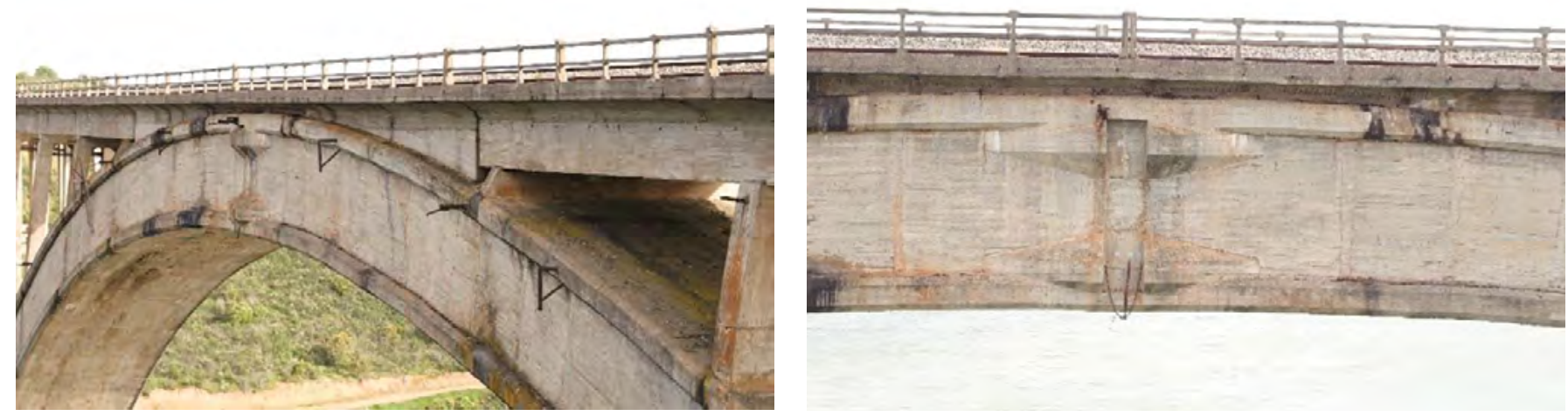

Figura 10. Vista general y de detalle de la zona de la clave del arco central del viaducto Martín Gil. (Fuente: Ineco). 


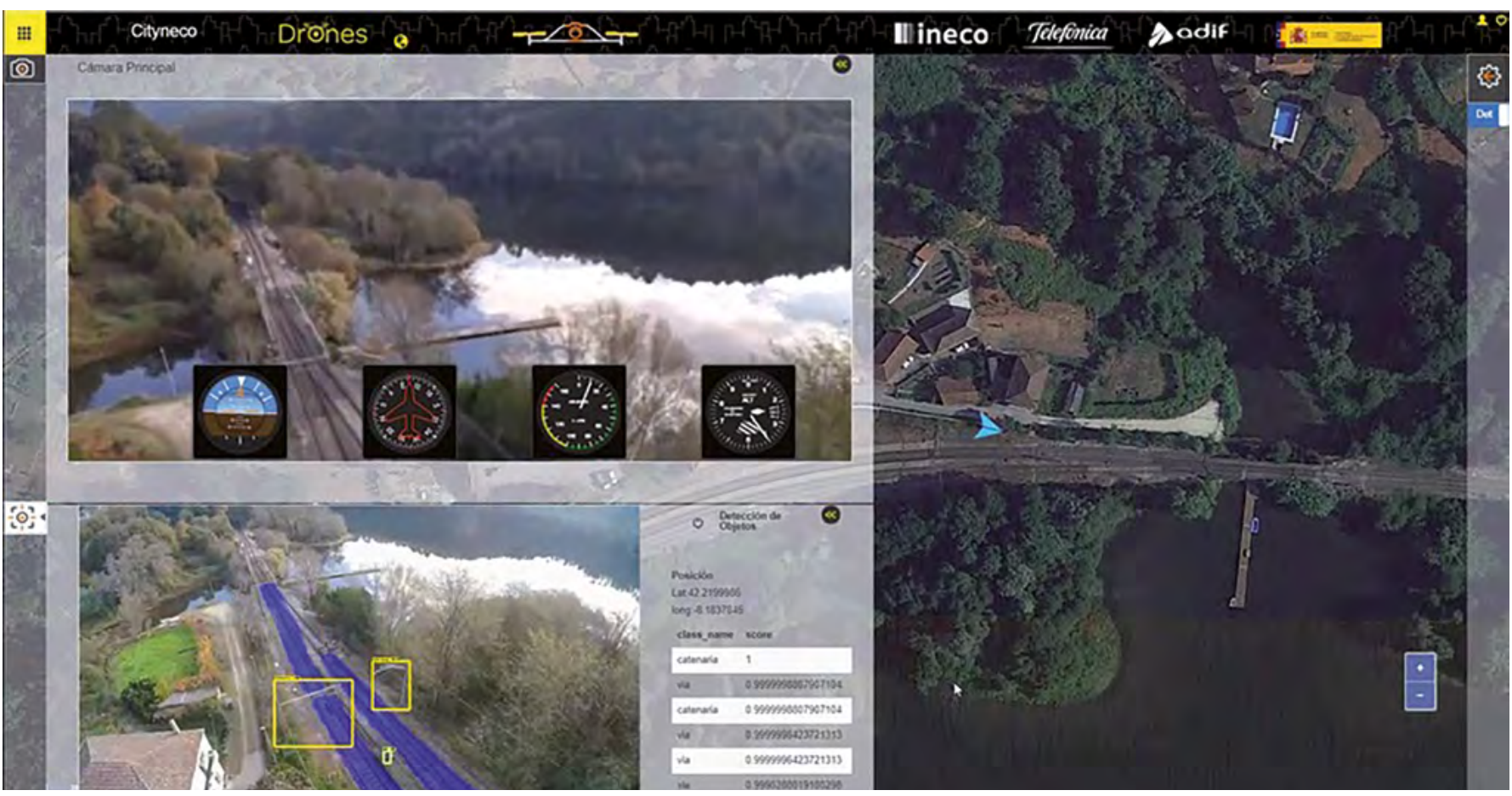

Figura 11. Vista del vuelo de prueba realizado con la colaboración de las empresas Telefónica, Huawei, Ineco y Adif.

La implantación de esta tecnología permitirá la inspección a distancia en tiempo real, pudiéndose incluso pilotar el dron desde un puesto remoto.

\section{5.}

\section{CONCLUSIONES}

Se ha realizado un breve recorrido histórico en lo referente a las normativas de puentes ferroviarios, desde el punto de vista de los aspectos relativos al mantenimiento y conservación de las estructuras, llegando hasta la situación actual. Se ha analizado con detalle el caso de las inspecciones básicas para comprender la necesidad de homogeneizar y estructurar este tipo de inspecciones, a fin de obtener resultados útiles para los gestores de las infraestructuras.

Se ha destacado la necesidad de extender la vigilancia de los puentes ferroviarios más allá de las inspecciones de carácter visual, haciendo uso de las nuevas tecnologías en materia de auscultación y monitorización de estructuras. Todo ello, nos conduciría a la necesidad de actualizar el marco normativo vigente, con la difícil tarea de aunar una regulación detallada de las actividades de vigilancia del estado estructural de los puentes ferroviarios y que esta sea compatible con la innovación y el desarrollo de nuevas técnicas y metodologías, de forma que se puedan garantizar los niveles de seguridad exigidos por la sociedad actual, optimizando económicamente los recursos disponibles.

\section{Referencias}

[1] Agencia Estatal Boletín Oficial del Estado (2005) Orden FOM/1951/2005 del 10 junio de 2005 por la que se aprueba la Instrucción sobre las Inspecciones Técnicas en los Puentes de Ferrocarril (ITPF-05).

[2] Consejo de Obras Públicas (1902) Instrucción para redactar los proyectos de puentes metálicos. Publicado en la Gaceta de Madrid - Num. 156 de 5 de junio de 1902.

[3] Consejo de Obras Públicas (1925) Instrucción para la redacción de proyectos de tramos metálicos. Publicado en la Gaceta de Madrid - Num. 297 de 24 de octubre de 1925.

[4] Agencia Estatal Boletín Oficial del Estado (1956) Orden de 17 de julio de 1956 por la que se aprueba la nueva Instrucción para el cálculo de tramos metálicos y previsión de los efectos dinámicos de las sobrecargas en los de hormigón armado. Publicado en el Boletín Oficial del Estado del 21 a agosto de 1956.

[5] Ministerio de Obras Públicas (1975) Instrucción relativa a las acciones a considerar en el proyecto de puentes de ferrocarril. Aprobada por la Orden ministerial de 25 de junio de 1975.

[6] Comisión Europea (2014) Reglamento (UE) No 1299/2014 de la Comisión de 18 de noviembre de 2014 relativo a las especificaciones técnicas de interoperabilidad del subsistema «infraestructura» en el sistema ferroviario de la Unión Europea

[7] Adif (2020) NAP 2-4-0.0_1E Norma Adif Plataforma. Inspección básica de puentes de ferrocarril. Aprobada en el comité de normativa del 29 de enero de 2020

[8] Adif (2020) NAP 2-4-1.0 Norma Adif Plataforma Inspección principal de puentes de ferrocarril. Aprobada en el comité de normativa del 22/07/2020

[9] Adif (2021) NAP 2-4-2.0 Norma Adif Plataforma Prueba de carga de puentes de ferrocarril (PC). Aprobada en el comité de normativa del 22/01/2021.

[10] Adif (2021) NAP 2-4-1.4 Norma Adif Plataforma Inspección principal de pasos superiores. Aprobada en el comité de normativa del 22/01/2021.

[11] Adif (2020) Criterios generales de mantenimiento preventivo de la especialidad de infraestructura y via.

[12] European Railway Operting Community (2014) Rail Technical Strategy Europe (RTSE). January 2014. ISBN: 978-2-7461-2269-7. 